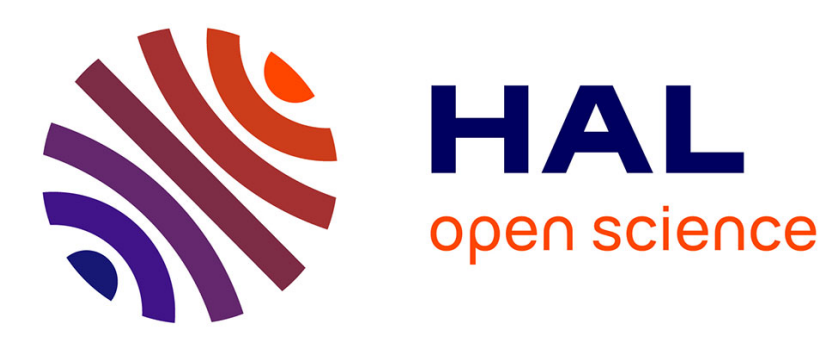

\title{
Conversion gain and noise in a Josephson mixer
}

Y. Taur, J.H. Claassen, P.L. Richards

\section{To cite this version:}

Y. Taur, J.H. Claassen, P.L. Richards. Conversion gain and noise in a Josephson mixer. Revue de Physique Appliquée, 1974, 9 (1), pp.263-268. 10.1051/rphysap:0197400901026300 . jpa-00243751

\section{HAL Id: jpa-00243751 https://hal.science/jpa-00243751}

Submitted on 1 Jan 1974

HAL is a multi-disciplinary open access archive for the deposit and dissemination of scientific research documents, whether they are published or not. The documents may come from teaching and research institutions in France or abroad, or from public or private research centers.
L'archive ouverte pluridisciplinaire HAL, est destinée au dépôt et à la diffusion de documents scientifiques de niveau recherche, publiés ou non, émanant des établissements d'enseignement et de recherche français ou étrangers, des laboratoires publics ou privés. 


\title{
CONVERSION GAIN AND NOISE IN A JOSEPHSON MIXER (*)
}

\author{
Y. TAUR, J. H. CLAASSEN and P. L. RICHARDS \\ Department of Physics, University of California, Berkeley, California 94720, USA
}

\begin{abstract}
Résumé. - Nous avons obtenu des rendements de conversion compris entre 0,33 et 4 et des températures de bruit de l'ordre de $200 \mathrm{~K}$, pour des mélangeurs constitués par des contacts à pointes non hystérétiques, et fonctionnant à $36 \mathrm{GHz}$. Ces rendements de conversion sont en bon accord avec le modèle de la jonction shuntée par une résistance. On rend compte du bruit observé, en supposant que le bruit Johnson de la résistance shunt, est la seule source de bruit. Quand le mélangeur est couplé à un système résonnant, le bruit est un peu plus grand que lorsqu'il est polarisé par une source de courant rf. Le mélange avec la fréquence fondamentale dans les jonctions hystérétiques est limité à une bande IF étroite, et donne un gain de conversion plus grand, mais aussi un bruit plus grand. Une amplification paramétrique à la fréquence IF est mise en évidence dans les jonctions hystérétiques. On a obtenu le mélange du quatrième harmonique de $9 \mathrm{GHz}$ avec des performances presque aussi bonnes que pour la fréquence fondamentale. Le mélange par couplage des modes d'une cavité apparaît moins prometteur.
\end{abstract}

\begin{abstract}
Fundamental mixing at $36 \mathrm{GHz}$ in non-hysteretic $\mathrm{Nb}$ point contacts has been observed with overall conversion efficiencies in the range $0.33 \leqslant \eta \leqslant 4$ and mixer noise temperatures in the neighborhood of $T_{\mathrm{M}} \approx 200 \mathrm{~K}$. The observed conversion efficiencies are in good agreement with the Resistively Shunted Junction model. The observed noise can be accounted for by assuming that the Johnson noise in the shunt resistor is the only source of noise driving the junction. Somewhat larger noise is seen from resonantly coupled mixers than is seen when the mixer is driven from an $\mathrm{rf}$ current source. Fundamental mixing in hysteretic junctions is limited to a narrow if bandwidth and gives much larger conversion gain but also larger noise. Parametric amplification at the if frequency is seen in hysteretic junctions. Fourth order harmonic mixing with $\omega_{\mathrm{LO}} / 2 \pi$ at $9 \mathrm{GHz}$ has been achieved with performance almost comparable to fundamental mixing. Cavity mode mixing appears less promising.
\end{abstract}

1. Introduction. - Point contact Josephson junctions have been suggested as low noise broadband nonlinear elements for millimeter wave devices [1]. The interpretation of early mixing experiments [2] was hampered by the absence of a detailed theoretical model for the junction response. The Resistively Shunted Junction (RSJ) model [3], assumes that a point contact can be represented by an ideal Josephson element, which carries pair current $I=I_{\mathrm{c}} \sin \varphi$, in parallel with a resistive shunt $R$. Computations based on this model have recently been found to be in excellent agreement with the static $I-V$ curves of oxidized $\mathrm{Nb}$ point contacts with and without a $36 \mathrm{GHz}$ microwave bias [4]. The effects of noise must be included in the calculation in order to obtain the correct differential resistance between steps [4], [5]. Very little change in the predicted junction properties is obtained if a phase modulated quasiparticle $(\cos \varphi)$ term is added to the RSJ model [6], [7]. This effect can therefore be neglected when calculating the performance of devices operated at frequencies below the energy gap.

Much of the interest in Josephson effect devices arises from the need for sensitive heterodyne receivers

(*) Research supported by the US Office of Naval Research. for frequencies above $100 \mathrm{GHz}$. Because of the difficulty of making accurate microwave measurements at such high frequencies we have set out to obtain a quantitative understanding of promising devices at $36 \mathrm{GHz}$ before making higher frequency measurements.

The waveguide structure shown in figure 1 has been used for our experiments. The oxidized $\mathrm{Nb}$ point contact junction is mounted in a thermally compensated structure [8] and is adjusted at room temperature by a $\mathrm{Nb}$ screw. The junction is ordinarily operated at the lowest temperature $4.2 \leqslant T<9 \mathrm{~K}$ for which there is no hysteresis on the static $I-V$ curve. The microwave radiation is resonantly coupled to the junction by means of a choke plunger and a tuning stub, both of which are adjustable at low temperature.

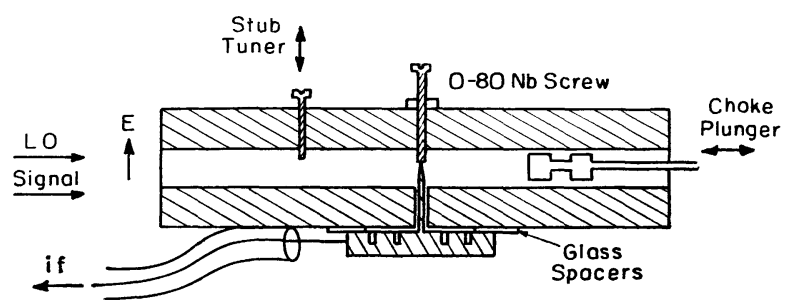

FIG. 1. - Josephson junction mixer. 
2. Microwave coupling. - In this work we have been primarily concerned with evaluating the performance of a heterodyne mixer with an external local oscillator. Consequently, our investigations of the matching of our junctions to a small rf signal power $P_{\mathrm{S}}$ have been carried out in the presence of a much larger local oscillator power $P_{\text {LO }}$. It is convenient to define a dimensionless parameter which is a measure of the coupling of the signal power to the junction,

$$
\alpha=\frac{\partial\left(I_{0} / I_{\mathrm{c}}\right)}{\partial\left[\left(8 P_{\mathrm{S}} / R I_{\mathrm{c}}^{2}\right)^{1 / 2}\right]}
$$

Here $I_{0}$ and $I_{\mathrm{c}}$ are the maximum zero voltage current with and without $\mathrm{rf}$ power and $P_{\mathrm{S}}$ is the available power from the small rf signal source. The value of $\alpha$ depends on the normalized frequency $\Omega=\hbar \omega_{\mathrm{S}} / 2 \mathrm{e} I_{\mathrm{c}} R$, the rf coupling, and $P_{\text {LO }}$.

Using published step height calculations from the RSJ model [4], [9] and assuming broad band coupling to the rf source, we can compute the maximum attainable value of $\alpha$ for a given $\Omega$. In figure 2 , we have plotted this theoretical maximum, along with the measured values of $\alpha_{\mathrm{opt}}^{2}$ that were obtained for a number of junctions (with and without hysteresis). The comparison between the experimental data and the calculated curve shows that nearly optimum coupling was achieved with high impedance junctions $(R \geqslant 20 \Omega)$, but the coupling was less good for low impedance junctions $(R \approx 5 \Omega)$. In the latter case the coupling was limited by microwave leakage. In figure 3 we plot the normalized rf source resistance $R_{\mathrm{S}} / R$ required to maximize $\alpha$. For small $\Omega$ the optimum source resistance is considerably smaller than has been previously assumed [10], [11].

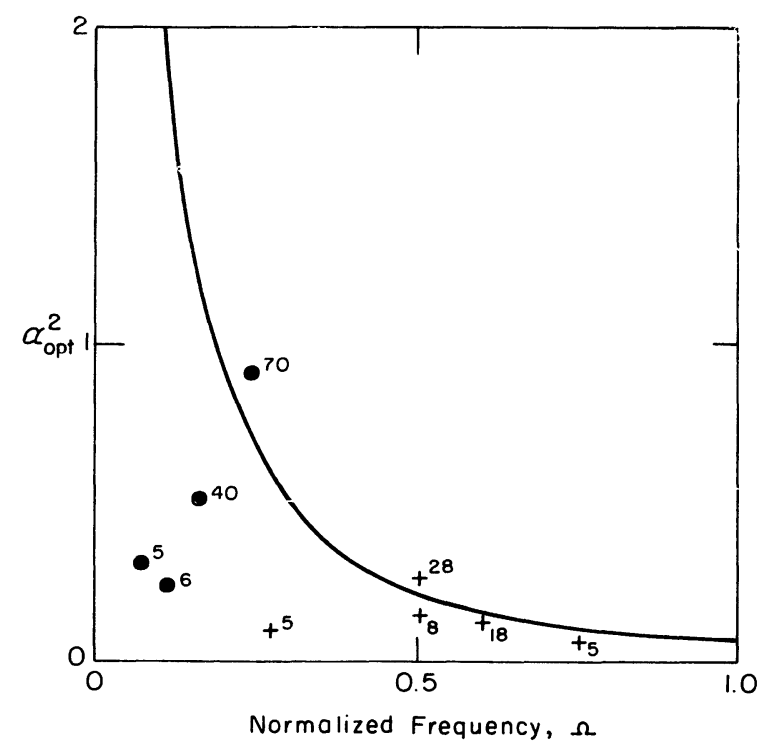

FIG. 2. - Measured optimum values of the parameter $\alpha^{2}$ for hysteretic (dots) and non-hysteretic junctions (crosses) compared with calculations from the RSJ model assuming broadband coupling. The numbers give the junction shunt resistance $R$ in ohms.

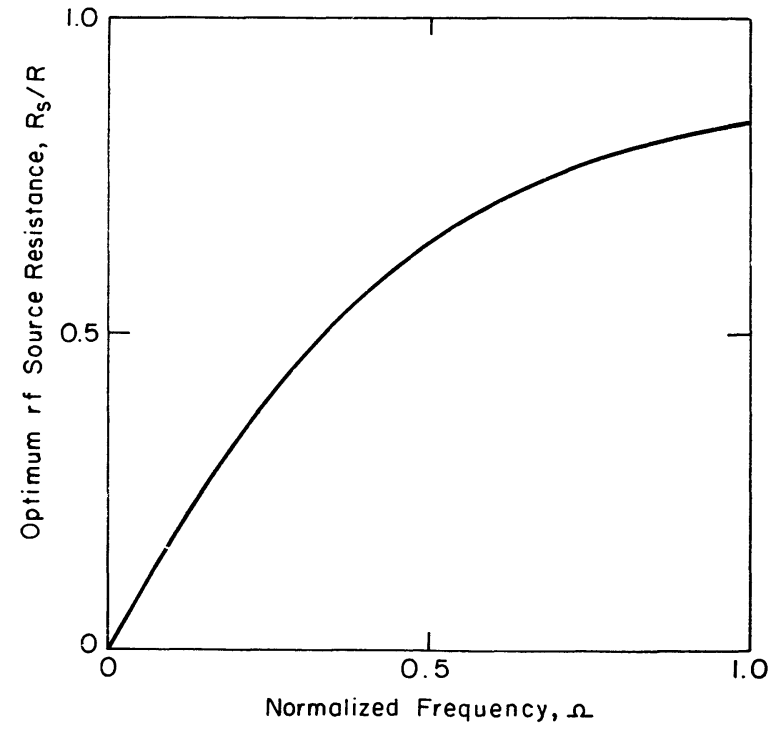

FIG. 3. - Optimum rf source resistance $R_{\mathrm{S}}$ computed from the RSJ model with broadband coupling, as a function of normalized frequency $\Omega$.

The shape of the static $I-V$ curve in the presence of rf power is expected from the RSJ model to depend on the rf source impedance. Since our junctions are resonantly coupled $(Q \lesssim 100)$ the source impedance depends on whether $\omega_{\mathrm{rf}}$ is greater or less than the cavity frequency $\omega_{\mathbf{c}}$. The experimental result is shown in figure 4.

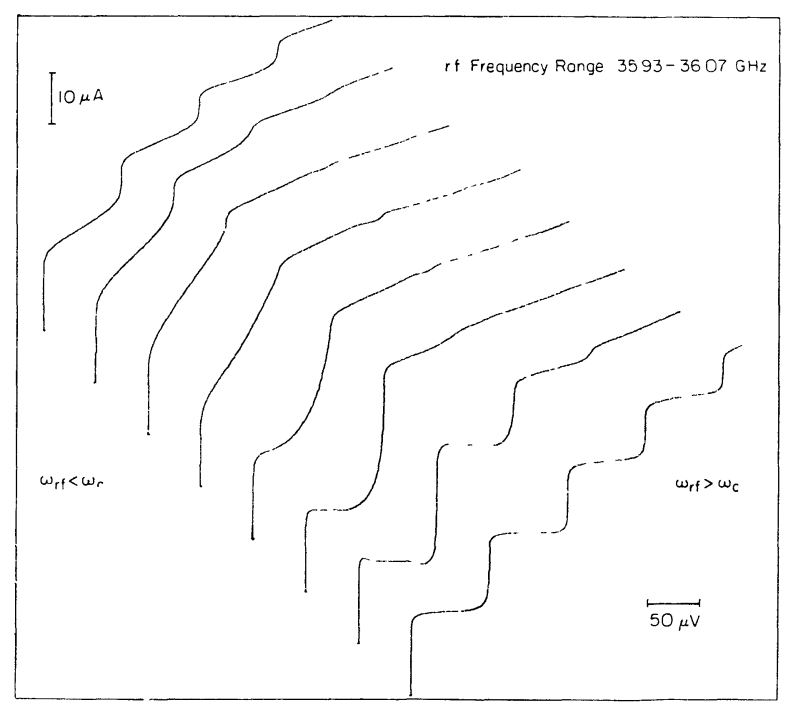

FIG. 4. - Changes in the static $I-V$ curves of a resonantlycoupled junction as the rf frequency is varied relative to the cavity frequency. The rf level was adjusted to produce the same depression of the critical current in each case, and was minimum for the fourth curve from the left, where $\omega_{\mathrm{rf}}=\omega_{\mathrm{e}}$.

We have used a Hamilton-type [12] junction simulator in order to compute this effect from the RSJ model. The resonant equivalent circuit used for the simulation is shown in figure 5 . The effect of the Johnson noise in $R$ is included by the use of a white noise 


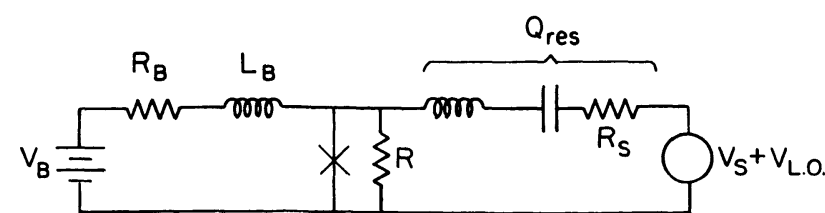

Fig. 5. - The equivalent circuit used with an analog junction simulator to compute the predictions of the RSJ model for a junction which is resonantly coupled to the rf source. The bias resistor $\boldsymbol{R}_{\mathrm{B}}$ serves as the if load, which is rf decoupled from the junction by the inductor $L_{\mathrm{B}}$.

source. The simulated $I-V$ curves shown in figure 6 are in excellent agreement with the data in figure 4. The simulator has also been used to show that, pro-

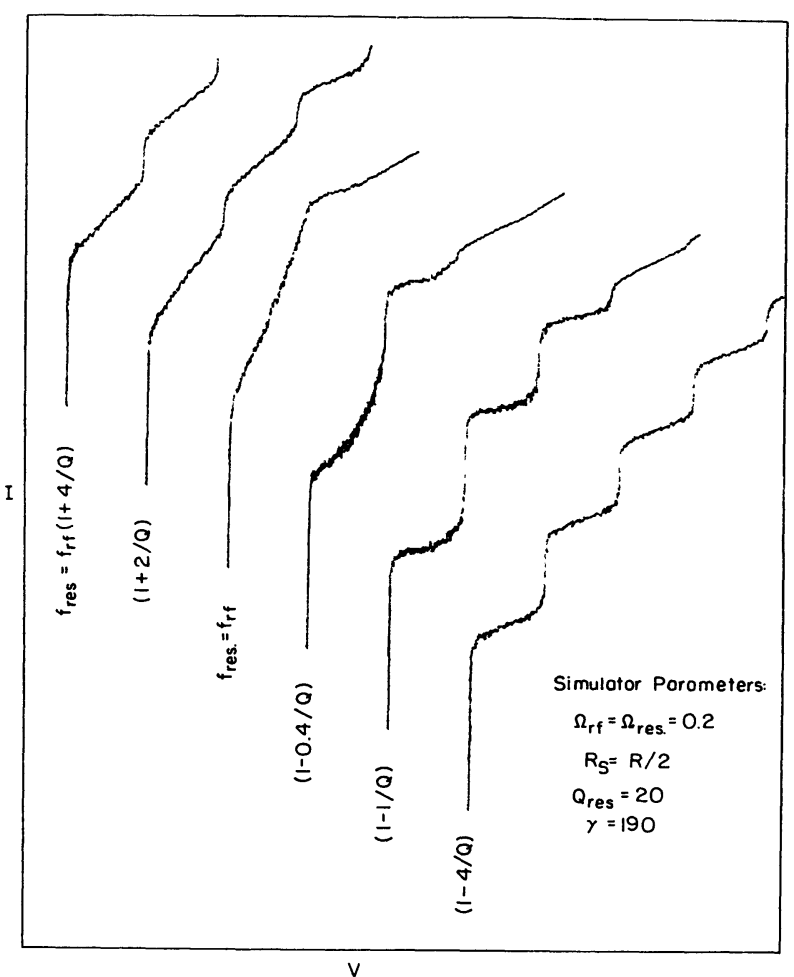

Fig. 6. - Analog simulation for conditions similar to those of figure 4. The parameter $\gamma=\hbar I_{\mathrm{c}} / \mathrm{e} k T$ characterizes the amount of noise that has been introduced into the simulator to represent Johnson noise.

vided $R_{\mathrm{S}}$ is not $\ll R$, the resonant source impedance does not significantly affect the RSJ predictions for the dependence of $\alpha_{\mathrm{opt}}^{2}$ and $R_{\mathrm{S}} / R$ on normalized frequency. The results show that the curves shown in figures 2 and 3, which were computed for broadband coupling, are also relevant to the case of resonant coupling.

3. Fundamental mixing. - The overall conversion efficiency $\eta$ of a heterodyne mixer is defined as the ratio of the power $P_{\text {if }}$ delivered to the intermediate frequency amplifier to the available power $P_{\mathrm{S}}$ from the signal source. The value of $\eta=P_{\text {if }} / P_{\mathrm{s}}$ computed from the RSJ model for a resistively coupled junction [10] can be conveniently expressed as

$$
\eta=\frac{C_{\mathrm{if}} \alpha^{2} R_{\mathrm{dyn}}}{R},
$$

where $C_{\text {if }}$ is the output coupling efficiency and $R_{\mathrm{dyn}}$ is the inverse slope of the static $I-V$ curve at the bias point.

Measured values of $\eta$ for junctions with and without hysteresis are shown in figure 7 and in table I. In the

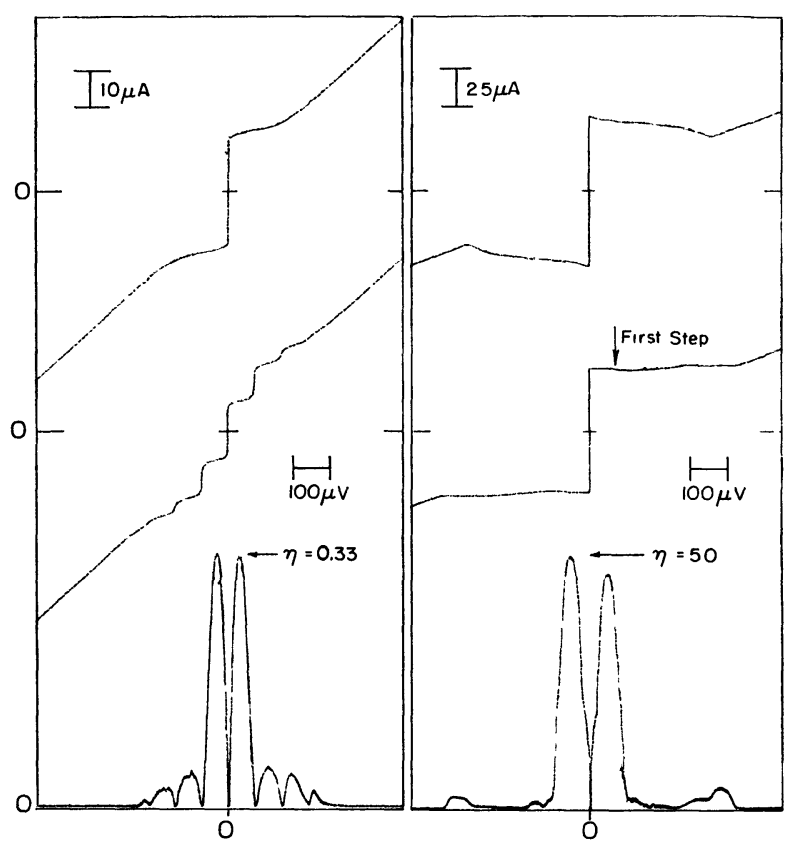

Fig. 7. - Static $I-V$ curves for $P_{\mathrm{LO}}=0$ and $P_{\mathrm{LO}} \neq 0$ and conversion efficiency for fundamental mixing as a function of bias voltage for two classes of junctions. The data on the left were obtained from a non-hysteretic junction operated at $T=8 \mathrm{~K}$ with the conversion efficiency measured at $20 \mathrm{MHz}$. The $I-V$ curve with $P_{\text {LO }}=0$ on the right shows negative resistance structure indicating a junction that would be hysteretic if biased by a current source. It was operated at $T=4.2 \mathrm{~K}$ with $\omega_{\text {if }} / 2 \pi=5 \mathrm{MHz}$. For both junctions the LO power required for maximum conversion efficiency was $P_{\text {LO }} \approx 10^{-9} \mathrm{~W}$.

absence of hysteresis, where the above theory is expected to apply, the peak values of $\eta$ were typically 70 to $90 \%$ of $C_{\text {if }} \alpha^{2} R_{\text {dyn }} / R$ in good agreement with eq. (2). The useful if bandwidth extends well beyond $100 \mathrm{MHz}$.

The very large conversion efficiency of hysteretic junctions is restricted to small bandwidths $\omega_{\text {if }} / 2 \pi \lesssim 5 \mathrm{MHz}$ and is associated with relaxation oscillations in the junction in this frequency range. Hysteretic junctions act as one port amplifiers for $\approx 5 \mathrm{MHz}$ signals introduced into the if cable. Gains of several hundred have been achieved for $P_{\mathrm{LO}}=0$, but the gain is reduced for $P_{\mathrm{LO}} \neq 0$. 
TABLE I

Summary of mixing results

\begin{tabular}{|c|c|c|c|c|c|c|c|c|}
\hline Mode of operation & $R$ (ohms) & $\Omega$ & $\alpha^{2}$ & $\underline{\eta}$ & $\underline{\beta^{2}}$ & $T_{\mathrm{N}}(\mathrm{K})$ & $T_{\mathrm{M}}(\underline{\mathrm{K}})$ & $T_{\text {AMB }}(\mathrm{K})$ \\
\hline $\begin{array}{l}\text { Fundamental mixing (no } \\
\text { hysteresis) }\end{array}$ & 28 & 0.5 & 0.23 & 0.33 & 5.0 & 80 & 200 & 8 \\
\hline & 5 & 0.27 & 0.1 & 1.0 & 4.5 & 180 & 170 & 4.2 \\
\hline & 5 & 0.5 & 0.18 & 4.0 & 6.5 & 1500 & 300 & 8 \\
\hline $\begin{array}{l}\text { Fundamental mixing (hys- } \\
\text { teresis) }\end{array}$ & 11 & 0.13 & & 50 & & $5 \times 10^{4}$ & 1000 & 4.2 \\
\hline Fourth harmonic mixing & 7 & 0.8 & 0.1 & 0.5 & 5.7 & 230 & 400 & 7 \\
\hline Cavity mode mixing & 1.6 & 0.5 & & $\approx 0.006$ & & & & 8 \\
\hline
\end{tabular}

4. Harmonic mixing. - Fourth order harmonic mixing has been achieved in the apparatus shown in figure 1 by introducing $9 \mathrm{GHz}$ local oscillator power via the coaxial cable. The static $I-V$ curves and the conversion efficiency are shown in figure 8 . The harmonic mixer performance summarized in table $I$ is nearly as good as for fundamental mixing. If this result remains valid at higher frequencies, it will greatly alleviate the problem of providing local oscillators for heterodyne mixers with signal frequencies beyond $100 \mathrm{GHz}$.

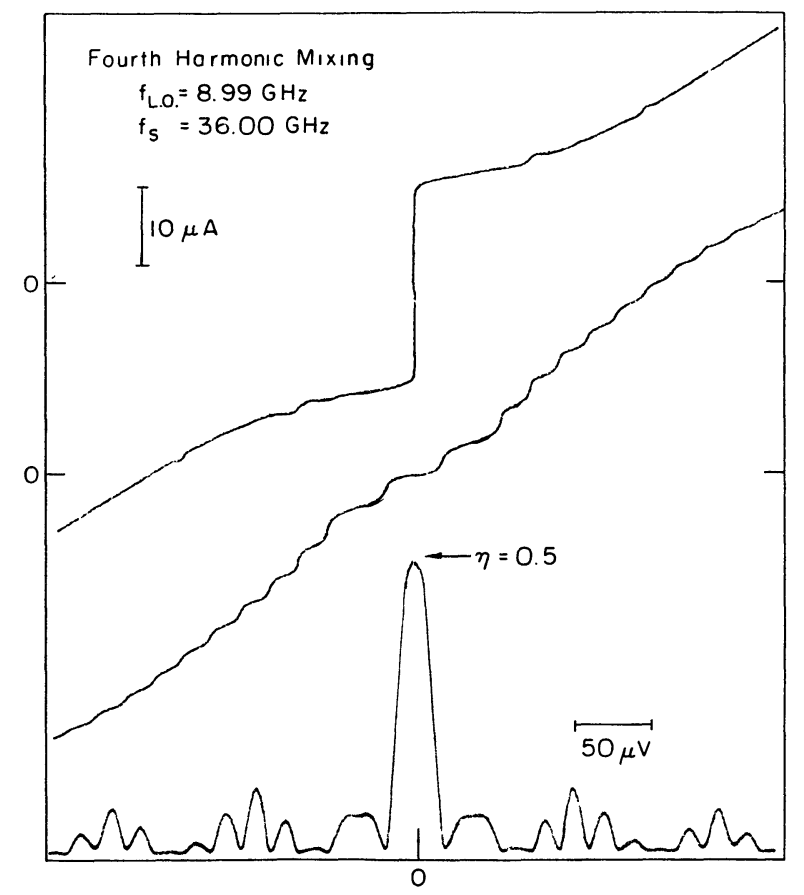

mode step on the junction $I-V$ curve at a bias voltage which corresponds to the cavity resonance. When the junction is dc biased near the cavity mode step it can be operated as a heterodyne receiver in which the ac Josephson oscillation, synchronized by the resonant cavity, serves as the local oscillator. We have measured the if response of a point contact operated in this mode. The junction $I-V$ curve as well as the conversion efficiencies for several different if frequencies are plotted in figure 9. We do not fully understand these complicated effects and so do not expect that the conversion efficiency has been optimized. The measured value of $\eta=0.006$, however, is not very encouraging.

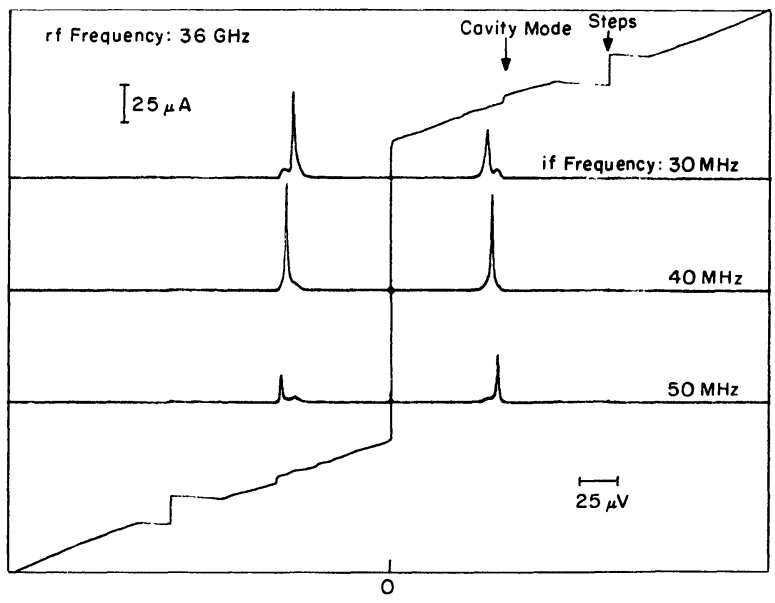

Fig. 9. - Frequency conversion using the Josephson oscillation synchronized to a cavity as LO. The output power in a $1 \mathrm{MHz}$ bandwidth is plotted as a function of bias voltage for three different if center frequencies, where the signal frequency is held constant. The corresponding junction $I-V$ curve is also shown.

Fig. 8. - Static $I-V$ curves for $P_{\mathrm{LO}}=0$ and $P_{\mathrm{LO}} \neq 0$ and conversion efficiency for fourth-order harmonic mixing. The maximum value of $\eta$ occurred at a value of $P_{\mathrm{LO}}$ which completely suppressed the zero voltage current.

5. Cavity mode mixing. - By minimizing the microwave leakage from the apparatus shown in figure 1 , it proved possible to produce a cavity temperature $T_{\mathrm{S}}$ referred to the input of a heterodyne mixer receiver can be written in terms of the noise temperatures of the antenna, the mixer, and the if amplifier,

$$
T_{\mathrm{S}}=T_{\mathrm{A}}+T_{\mathrm{M}}+T_{\mathrm{if}} / \eta .
$$


Eq. (3) illustrates how $T_{\mathrm{M}}$ and $\eta$ enter into the performance of a heterodyne system. To determine $T_{\mathrm{M}}$, we measure the effective junction noise temperature $T_{\mathrm{N}}$ and the if coupling efficiency $C_{\mathrm{if}}$, which are related to the mixer noise temperature by

$$
T_{\mathrm{M}}=T_{\mathrm{N}} C_{\mathrm{if}} / \eta
$$

Both $T_{\mathrm{N}}$ and $C_{\mathrm{if}}$ are obtained by substituting a resistor for the junction whose value is equal to the junction dynamic resistance. $T_{\mathrm{N}}$ is defined as the temperature of this resistor which produces the same output noise level from the if amplifier as was observed for the junction mixer with $T_{\mathrm{A}}=0 . C_{\mathrm{if}}$ is obtained by noting the rate of change of if output power with temperature of the resistor ; explicitly,

$$
C_{\mathrm{if}}=\mathrm{d} P_{\mathrm{n}} / \mathrm{d}\left(k_{\mathrm{B}} T\right),
$$

where $P_{\mathrm{n}}$ is the if noise power/bandwidth referred to the input. In table I we show values of $T_{\mathrm{N}}$ along with values of the mixer noise temperature $T_{\mathrm{M}}$ for various heterodyne mixers with external local oscillator. For fundamental mixing with non-hysteretic junctions, the values of $T_{\mathrm{M}}$ cluster around $200 \mathrm{~K}$.

7. Junction noise. - We believe that our measured mixer performance is limited by inherent junction fluctuations. The noise temperature $T_{\mathrm{N}}$ is expected to be proportional to the ambient temperature $T_{\mathrm{AMB}}$ and also to $R_{\mathrm{dyn}} / R$. We have made careful measurements of $T_{\mathrm{N}}$ for junctions driven by an rf current source. These are best presented in terms of a dimensionless parameter $\beta^{2}$ defined such that

$$
T_{\mathrm{N}}=\beta^{2} T_{\mathrm{AMB}} R_{\mathrm{dyn}} / R .
$$

Values of $\beta^{2}$ so defined turn out to be relatively insensitive to $R_{\mathrm{dyn}}$ and $T_{\mathrm{AMB}}$. Previous measurements [13] of noise in point contacts were compared with a theory for which $\beta^{2}=1$. In figure 10 we show static $I-V$ curves for $P_{\text {LO }}=0$ and $P_{\text {LO }} \neq 0$ along with the measured values of $\beta^{2}$. A linearized analytic theory of noise in the RSJ model [14] is available for the case $P_{\text {LO }}=0$. It assumes that the noise arises from ambient temperature Johnson noise in the shunt resistor and should be valid in the limit of no noise rounding of the $I-V$ curves. A curve for $\beta^{2}$ obtained from this theory is plotted in figure 10 along with the data for $P_{\mathrm{LO}}=0$. The observed values of $\beta^{2}$ are larger than the predicted ones. This is to be expected since the noise rounding of the experimental $I-V$ curves is quite severe so the small noise limit assumed in the theory is not valid.

In order to test whether the observed noise actually arises from Johnson noise in $R$, we have made noise measurements with the junction simulator for the experimental situation of considerable noise rounding. The results of our simulation are shown in figure 11 .
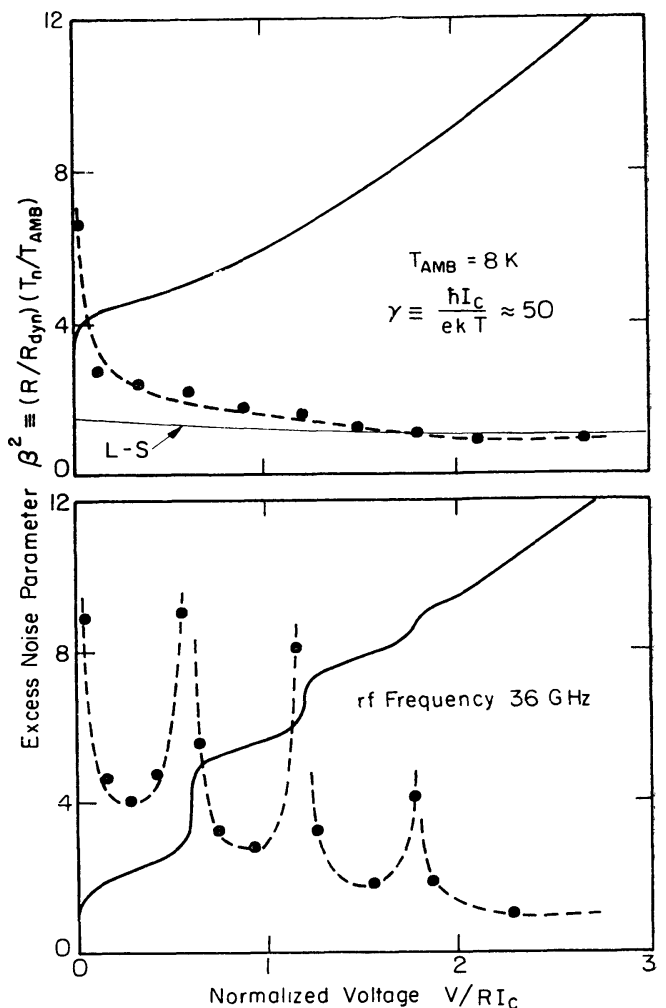

FIG. 10. - Measurements of the noise parameter $\beta^{2}$ as a function of bias voltage with $P_{\text {LO }}=0$ and $P_{\text {LO }} \neq 0$, with the corresponding $I-V$ curves. The coupling of this junction to the $\mathrm{rf}$ source was intentionally poor. The light curve gives the results of the linearized noise theory of Likharev and Semenov. It is not expected to be valid for such small values of the parameter $\gamma$.

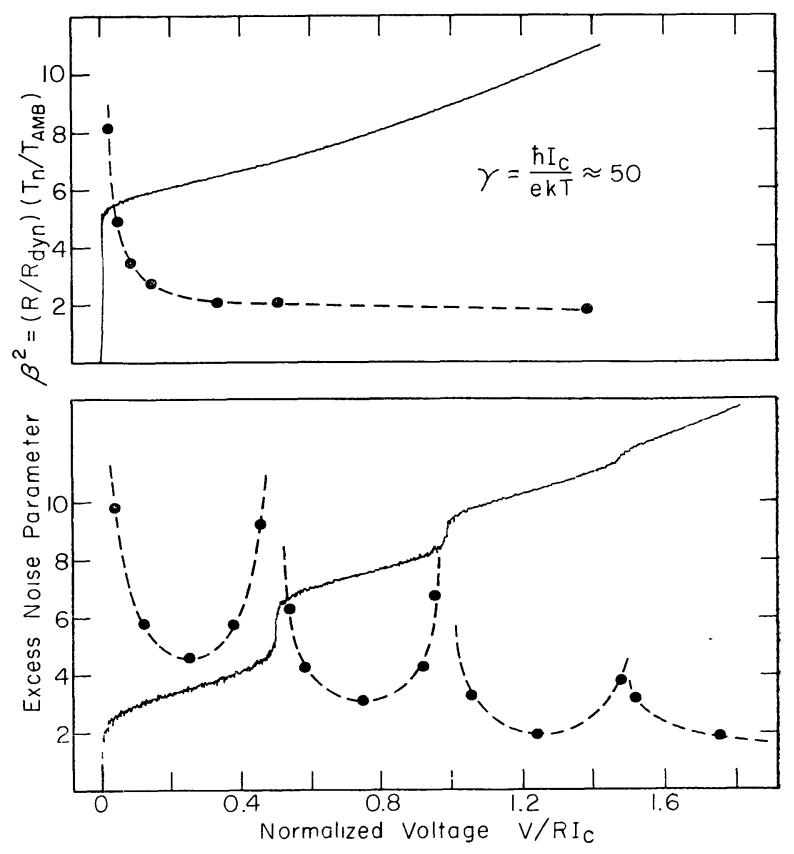

FIG. 11. - Calculated values of the noise parameter $\beta^{2}$ as determined by the simulator along with the corresponding $I-V$ curves, for conditions similar to those of the real junction in figure 10. Evidently the agreement is excellent. 


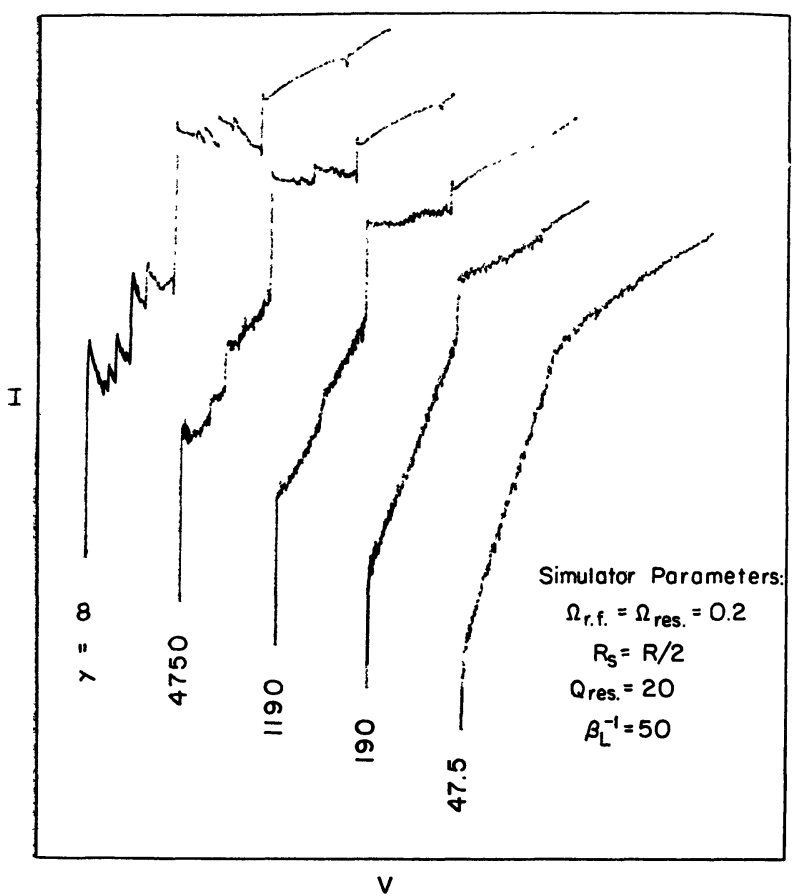

FIG. 12. - Simulated static $I-V$ curves for a resonantly matched junction as a function of $\gamma=\hbar I_{\mathrm{c}} / \mathrm{e} k T$. The simulated biasing inductor, characterized by $\beta_{\mathrm{L}}=\hbar / 2 \mathrm{e} L_{\mathrm{B}} I_{\mathrm{c}}$, has been made rather small to reveal the cavity mode steps which appear for large $\gamma$ (small noise).
They are remarkably similar to the noise observed from the real junction.

Values of $\beta^{2}$ measured for junctions used as resonantly coupled rf mixers are given in table $\mathrm{I}$. These values are somewhat larger than those shown in figure 10 for junctions driven with an rf current source. This same effect is seen in the resonantly coupled junction simulator. It appears possible, therefore that the resonant rf coupling is introducing excess noise despite the fact that the feedback is too weak to overcome the thermal fluctuations and synchronize the junction. By using the simulator we can reduce the effective ambient temperature to values well below those available in the actual experiment. The simulations in figure 12 show that a multitude of cavity mode steps appear in an apparently smooth $I-V$ curve when the noise is reduced. We may speculate that the excess noise which appears in our resonantly coupled mixers is associated with these incompletely averaged cavity modes. It appears probable that the performance of some of our mixers could be improved by the use of non-resonant rf coupling structures.

Acknowledgments. - The authors are grateful to the IMRD, Lawrence Berkeley Laboratory for the use of their apparatus. The microwave choke filters were designed by B. Leskovar and D. Hopkins.

\section{References}

[1] For a recent review, see Richards, P. L., Auracher, F. and VAN DuZER, T., Proc. IEEE 61 (1973) 36.

[2] Grimes, C. C. and Shapiro, S., Phys. Rev. 169 (1968) 397.

[3] McCumber, D. E., J. Appl. Phys. 39 (1968) 3113.

[4] Taur, Y., Richards, P. L. and Auracher, F., Proceedings of the Thirteenth International Conference on Low Temperature Physics, 1972.

[5] Henkels, W. H. and WebB, W. W., Phys. Rev. Lett. 26 (1971) 1164

[6] Auracher, F., Richards, P. L. and Rochlin, G. I., Phys. Rev. (to be published).

[7] Falco, C. M., Parker, W. H. and Trullinger, S. E. (to be published)
[8] Buhrman, R. A., Strait, S. F. and Webb, W. W., Technical Report \# 1555, The Material Science Center, Cornell University (1971).

[9] Russer, P., J. Appl. Phys. 43 (1972) 2008.

[10] Auracher, F. and Van Duzer, T., Applied Superconductivity Conference (1972).

[11] Kanter, H. (to be published).

[12] Hamilton, C. A., Rev. Sci. Instrum. 43 (1972) 445.

[13] Kanter, H. and Vernon, F. L., Jr., Phys. Rev. Lett. 25 (1970) 588.

[14] Likharev, K. K. and Semenov, V. K., Sov. Phys. JeTP 15 (1972) 442. 